\title{
TERAPIA OCUPACIONAL EN EL HOSPITAL: CONSTRUCCIONES DE SENTIDO EN UNA COMUNIDAD DE PRÁCTICA
}

\author{
OCCUPATIONAL THERAPY AT THE HOSPITAL: MEANING CONSTRUCTIONS ON A
} COMMUNITY OF PRACTICE

\author{
Taís Quevedo Marcolino', Regina Helena Vitale Torkomian Joaquim², Tatiana Barbieri \\ Bombarda $^{3}$ y Aline Maria de Medeiros Rodrigues Reali ${ }^{4}$
}

\section{Resumen}

En el hospital, los pacientes no tienen control sobre su tiempo, lo que les puede ocasionar situaciones que pongan en riesgo la vida. En una investigación-acción realizada para comprender el desarrollo profesional y el razonamiento clínico de terapeutas ocupacionales principiantes construidas como Comunidad de Práctica (CoP), se realizaron 18 encuentros presenciales, grabados, transcritos y sometidos a análisis temático. Los resultados de la subcategoría temática "práctica en el hospital y tensiones con el modelo biomédico", indicaron cómo la tensión con el modelo biomédico se hace presente en las construcciones de la CoP, en la medida en que al adentrar en el mundo cóno la tension con el mo clinico y no-cotidiano del hospital, la terapia ocupacional fue llamada a ofrecer posibilidades de vida, favoreciendo que los pacientes se ubiquen de modo diferente en la vida y logren ocupar el tiempo vacio y comun en la espera por procedimientos con su creatividad personal, favoreciendo (a) el cuidado clínico de la enfermedad (b) una construcción - que puede ser compartida con otras personas del equipo - de la imagen de una persona que hace, piensa y tiene una historia de vida, que no solamente ocupa el lugar de enfermo, (c) un mejor manejo del dolor y de los procedimientos hospitalarios; tal como (d) procedimientos en la clínica de la terminalidad. Además, el ejercicio de investigar la práctica y de compartirla en un espacio formativo, como la CoP, permitió la apropiación del razonamiento clínico a partir de las particularidades de una práctica de terapia ocupacional en contexto hospitalario.
\end{abstract}

\section{Palabras clave}

Terapia ocupacional, Práctica profesional, Narración, Hospitalización.

Mestre e Doutora em Educação pela Universidade Federal de São Carlos. Terapeuta ocupacional pela Universidade Federal de São Carlos. Rodovia Washington Luiz, km 235, São Carlos, SP, Brazil, CEP 13565-905, taisquevedo@gmail.com, +55 16 33518111. Researcher ID C-81102014 ORCID 0000-0002-9694-5118

Mestre e Doutora em Educação Especial pela Universidade Federal de São Carlos. Terapeuta ocupacional pela Universidade Federal de São Carlos. Rodovia Washington Luiz, km 235, São Carlos, SP, Brazil, CEP 13565-905, joaquimrhvt@gmail.com, +55 1633518111 . ORCID 0000-0003-3700-397X

Mestre em Terapia Ocupacional pela Universidade Federal de São Carlos. Terapeuta ocupacional pela Universidade Sagrado Coração Rodovia Washington Luiz, km 235, São Carlos, SP, Brazil, CEP 13565-905, tatibb_to@yahoo.com.br , +55 16 33518111. ORCID 0000-00029478-7945

4 Mestre em Educação Especial pela Universidade Federal de São Carlos, Doutora em Psicologia pela Universidade de São Paulo. Psicóloga pela Universidade de São Paulo, Rodovia Washington Luiz, km 235, São Carlos, SP, Brazil, CEP 13565-905, alinereali@gmail.com , +55 16 33518111.ORCID 0000-0003-4915-8127 


\begin{abstract}
In the hospital, patients have no control over their time, possible standing near diseases that endanger life. In an action research focused to understand professional development and clinical reasoning of beginner occupational therapists, built as a Community of Practice (CoP) 18 person meetings were transcribed and submitted to thematic analysis. Results of subcategory "practice in hospital and stress to the biomedical model" shown as the tension with the biomedical model was present in constructs of the CoP in that when occupationa he biomedic ling differenty and the cliticher

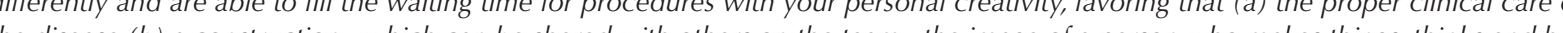

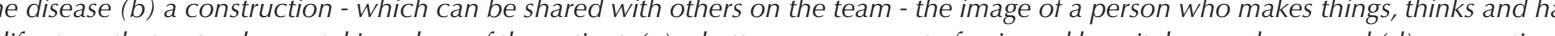
a life story, that not only one taking place of the patient, (c) a better management of pain and hospital procedures; and (d) occupational therapy procedures for terminal patients. In addition, exercise to investigate the practice and to share it in a formative space, such as the CoP, favored the clinical reasoning appropriation on peculiarities of the practice of occupational therapy in the hospital setting.
\end{abstract}

\title{
Keywords
}

Occupational therapy, Professional practice, Narrative, Hospitalization

Recepcionado: 16/11/2016

Aceptado: 09/06/2017

\section{INTRODUCCIÓN}

A partir de The Boston Clinical Reasoning Study, investigación realizada a finales de la década de 1980 en un hospital general de la ciudad de Boston, en los Estados Unidos, el conocimiento práctico en Terapia Ocupacional ha sido investigado desde la perspectiva de razonamiento clínico y profesional (Mattingly \& Fleming 1994), indicando que el pensamiento narrativo, aque orientado al mundo humano de los motivos, valores y creencias (Bruner, 1997), sustenta el proceso terapéutico.

Desde una perspectiva fenomenológica (Mattingly \& Fleming, 1994), el hospital fue comprendido en dos espacios-tiempo. El primero, expresado en el tiempo de médico, rápido e eficiente, cuyo héroe es el médico en su batalla contra la enfermedad; y el segundo, el de los otros profesionales de la salud, que se mueven más despacio y que pasan más tiempo con los pacientes, muchas veces en situaciones informales (Mattingly, 1998). El terapeuta ocupacional transita en ese segundo espacio-tiempo $y$, sin embargo ofrece cuidados del orden biomédico desde la recuperación, rehabilitación y prevención o secundaria a los daños (Palm, 2016) demanda que los pacientes asuman otro lugar, estimulándolos a ser $\mathrm{CO}^{-}$ -participes en el proceso de internación, dan el fomento a la adquisición de informaciones sobre la enfermedad y los procedimientos del tratamiento, y a la construcción de un modo particular de estar en el hospital a partir de sus necesidades cotidianas y ocupacionales (Álvarez et al, 2011; Palm, 2016; Riffo, 2013).
En el hospital hay una tendencia a la despersonificación (Angerami-Camon, 2010), los pacientes no tienen control sobre su tiempo, siendo sometidos a la pasividad restrictos a espacios, manifestaciones y al contacto con su ambiente socio-familiar (Angeli, Luvizaro \& Galheigo 2012). Al pretender construir con los pacientes experiencias significativas que los ubiquen de un modo diferente en sus vidas, los terapeutas ocupacionales invitan a ser héroes narrativos, a aquellos que deben luchar las batallas (Mattingly, 1998).

En ese trabajo, se percibe una tensión inherente a la terapia ocupacional, pues el tiempo narrativo, que es un tiempo de transformación, caracterizado por la vivencia de los eventos y por la centralidad del actor, y no de la enfermedad y de su tiempo cronológico, es poco valorizado en el mundo biomédico, llevando a terapeutas ocupacionales a no siempre explicitar su trabajo fenomenológico, asumiendo públicamente un discurso exclusivo de tratamiento de déficits funcionales (Mattingly \& Fleming, 1994; Pierre, 2009; Robertson, 2012; Schell \& Schell, 2008)

Además, en el hospital se vive la constante fragilidad en sufrir daños que ponen en riesgo la vida, incluso la muerte, pues está hoy desplazada para el espacio hospitalario (Briceño, 2011). Esta fragilidad abre un espacio potencial de creación para los profesionales, en especial terapeutas ocupacionales, en su lucha por hacer existir un cotidiano del sujeto en el mundo no-cotidiano de la 
clínica (Mattingly, 1998), y para los propios pacientes, que pueden construir nuevos sentidos sobre sus vidas en este momento.

De este modo, en una investigación-acción interesada en el desarrollo profesional y del razonamiento clínico de terapeutas ocupacionales principiantes, este aspecto de la práctica se hizo presente. Este artículo busca discutir las construcciones de sentido en esa temática, en una Comunidad de Práctica.

\section{METODO}

En el estudio se utilizó la investigación-acción participativa (Desroche, 2006; Toledo \& Jacobi, 2014), modalidad cualitativa que busca tanto la producción de conocimientos sobre la práctica como su perfeccionamiento.

La investigación-acción fue construida como Comunidad de Práctica (CoP), por caracterizarse como un emprendimiento colectivo, en torno a un objetivo común, pautado en la negociación de significados por la participación de todos los sujetos incluidos y por la concretización (lo que va siendo producido) de esa participación (Marcolino et al., 2016; Galheigo et al, 2015; Wenger, 1998).

Una CoP ofrece oportunidades para que sus miembros reflexionen, dialoguen, cuestionen y compartan sus conocimientos y recursos. Las soluciones y comprensiones que emerjan de las interacciones entre los participantes generan un "cúmulo de conocimiento" que es incrementado con el pasar del tiempo y se incorpora a la práctica. A través de la interacción entre los miembros, el conocimiento tácito se torna explícito, creando relacionamientos interpersonales basados en la confianza (Preece, 2004; Wenger, 1998; Wesley \& Buysse, 2001).

El objetivo común de la CoP fue comprender el proceso del desarrollo profesional y del razonamiento clínico de terapeutas ocupacionales principiantes. Los participantes fueron seis terapeutas ocupacionales principiantes (cursando una especialización en Terapia Ocupacional en Salud Mental); dos terapeutas ocupacionales experimentadas (profesoras del referido curso), y la investigadora (coordinadora de la CoP). La CoP existió por diez meses (marzo a diciembre de 2007), en dieciocho encuentros presenciales, con aproximadamente una hora, y frecuencia quincenal, grabados en audio y transcritos (Marcolino \& Reali, 2016). En la CoP, las profesionales principiantes compartieron y reflexionaron sobre sus prácticas, y también mantuvieron un diario reflexivo compartido con la investigadora-coordinadora, favoreciendo una constante interacción entre lo que era producido en los encuentros presenciales de la CoP y en los diarios (Marcolino et al, 2017).

Las transcripciones de los encuentros presenciales fueron sometidas al Análisis Temático (Bardin, 2011), identificándose tres grandes categorías, a saber: 1. la construcción en la investigación-acción; 2 . ser terapeuta ocupacional principiante 3 . la asistencia en terapia ocupacional. La tercer categoría fue sometida a nuevo análisis temático y, en ese nuevo proceso, se identificaron tres subtemas: (a) el discurso público de terapia ocupacional, (b) la práctica en el hospital y las tensiones con el modelo biomédico y (c) el proceso terapéutico. Además, para comprender como esos contenidos fueron constituyéndose a lo largo del tiempo, se realizó un análisis de la línea de tiempo (Grossman, Winenburg \& Woolworth, 2001; Marcolino \& Reali, 2012).

En este artículo, serán presentados los resultados del subtema "Ia práctica en el hospital y las tensiones con el modelo biomédico" y su movimiento de construcción de sentidos a lo largo de la investigación-acción. Los nombres de los participantes son ficticios. La investigación fue aprobada por Comité de Ética del Centro Universitario de Araraquara, bajo el número 476. Las participantes firmaron el término de consentimiento informado para la investigación y autorizaron la publicación.

\section{RESULTADOS}

Las construcciones sobre la temática "las tensiones con el modelo biomédico en la a asistencia" sucedieron en el décimo primero y en el décimo segundo encuentro presencial, al compartir narrativas sobre la práctica de las participantes en el hospital. El diálogo pareció tomar una mayor profundidad cuando la asistencia en terapia ocupacional fue contrastada con del modelo biomédico, al explicitarse comprensiones y creencias sobre lo que es común, o canónico, del proceso terapéutico en terapia ocupacional y las particularidades de ese proceso en el contexto hospitalario.

Un primer elemento canónico puede ser encontrado en lo dicho por Isadora, en el décimo primer encuentro, cuando explicita su dificultad en trabajar con un paciente en estado terminal, pues está acostumbrada a una terapia ocupacional que trabaja con proyectos de vida.

"[...] estamos tan acostumbrados a hacer proyectos de vida, construir proyectos junto 
al paciente que la verdad, en ese caso, no tenemos como hacer proyectos, [...] un proyecto de muerte, como si fuese la última actividad [...]" (Isadora)

En el décimo segundo encuentro, al conversar sobre los dilemas del tiempo de tratamiento en el hospita general, en un servicio de Interconsulta en Salud Mental $^{5}$, aparece otro elemento que puede ser considerado canónico en un tratamiento de terapia ocupacional, po lo menos en la práctica en salud mental, que es de un período de tratamiento más largo, como en la clínica de la psicosis, de la psiquiatría.

"[...] el tiempo en la Interconsulta es bien diferente del tiempo [...] de la clínica en la psicosis [...]" (Luiza, décimo segundo encuentro)

"O del tiempo que estamos acostumbrados a tener en un tratamiento que pensamos [...] en la terapia ocupacional." (Clarice, décimo segundo encuentro)

Otra indicio de esa canonicidad puede ser observado cuando, en el décimo cuarto encuentro, la coordinadora propuso que las participantes imaginen una terapeuta ocupacional atendiendo. La descripción de estas imaginaciones mostró espacio físico y subjetivo común de trabajo del terapeuta ocupacional, un encuentro en una sala de atención con mesa, materiales y armarios.

\section{"[...] lo básico [...] es el encuentro [...] veo} la sala [...] dos personas [...]." (Marisa)

"Con un montón de cosas al rededor." (Fernanda)

"Y, armarios, materiales." (Marisa)

El espacio-tiempo vivido por los pacientes, con el poco control sobre su tiempo, viviendo en la constante espera (Mattingly, 1998), fue percibido con angustia pero como potencia para comprender la situación de los pacientes y para crear posibilidades de intervención en terapia ocupacional.

Se trata de un servicio en el cual un equipo de salud mental es accionada por equipos de otras Enfermerias en el Hospital Genera para actuar tanto junto a pacientes como junto al propio equipo.
"[...] yo creo que es un lugar que llama mucho la atención, [...] en un tiempo [... que no existe, que [...] es un vacío [...] es la espera, es la nada, [...]" (Mariana)

Al defender que terapia ocupacional implica vida, y que al ofrecer actividades, otro lugar es facilitado a los pacientes: de quien es cuidado (enfermo) para alguien más activo, que produce, que puede cuidar de otro, ofrecer al otro. Los pacientes se sienten más vivos, establecen otras relaciones, otros diálogos con los demás pacientes y con el equipo en un discurso que no es solo el de la enfermedad. El vacío de la espera, es rellenado por las posibilidades de construir algo, construir narrativas, ya sea una historia que esté casi terminando, y mejorando inclusive, el cuidado biomédico (acceder, lidiar con el dolor). Es el héroe narrativo que surge (Mattingly, 1998).

"[...] al ofrecer actividades, se ofrece otro espacio, se ofrece vida - que muchas veces no se tiene dentro de un hospital - y se ofrece otro papel también, [...] puede salir del papel de quien sólo es cuidado, de quien está todo el tiempo como enfermo, para tornarse alguien más activo, que produce, que puede cuidar del otro, que puede brindar al otro." (Clarice)

"[...] y el momento que tiene la posibilidad de construir algo [...] que remita a las historias, yo creo que usted construyó narrativas con ellos, [...] de alguna forma, es la actividad y la relación con el terapeuta, [...] traer un poco de lo que es de afuera, para así poder, [...] continuar alguna historia, aunque sea una historia que esté casi terminando, que, [...] está en una incerteza [...] hacer algo diferente, [...] es algo que los ayuda a unirse por un discurso que no sea solo el de la enfermedad, pero de 'ah, vos hiciste eso', 'ah, vos venís de ahi' [...] y el equipo que entra y ve y [...] habla 'mirá, pero vos hiciste eso?' [...] o, entonces [...] 'cuando ustedes (terapeutas ocupacionales) están ahí es más fácil poder acceder, [...] creo que eso del dolor, [...] no que no tenga el dolor pero creo que es un dolor diferente, que tal vez ya no sea tan solitario, [...]" (Mariana) 
En ese sentido, las actividades, que personifican los pacientes, parecen imprimir otras posibilidades dentro del hospital, en oposición a la despersonalización impuesta por el cuidado de la enfermedad.

"[...] la muerte estaba allá todo el tempo, [...] al mismo tiempo las internaciones prolongadas, [...] y los grupos, [...] era donde las personas se conocían de una u otra forma, [...] no era la cama tal, [...] tenía algo [...] de poder contar, [...] de la vida, [...] de lo que había sucedido hasta entonces, [...] de presentar la familia, lo que le gustaba, lo que no, $[\ldots]^{\prime \prime}$ (Tatiane)

"[...] es que muchos profesionales, [...] evitan eso porque zqué es lo que hacés cuando conocés a una persona que sabés que va a morir en poco tiempo? Lleva a mantener ese distanciamiento con el trasplantado [...] es defensivo, claro, [...] vos no entrás tanto en contacto con esa persona, con la vida de ella, con los proyectos de ella, que serán interrumpidos, [...] y la terapeuta ocupacional no tiene alternativa, $[. . .]^{\prime \prime}$ (Tatiane)

"[...] creo que es un equipo que pasa por muchos aprietos, [...] una carga de sufrimiento muy grande [...] y en cuanto que había una producción, porque creo que también es diferente [...] de ir para una conversación [...] aquel material queda y aquel material circula, [...] entonces el equipo que entra a la tarde, ve y pregunta 'pero iquién hizo, con quien, donde hizo?', entonces eso parece que va dando otra vida también, va teniendo una circulación." (Mariana)

"Que no es solo en aquel tiempo, parece que perdura un poco más, [...] muy interesante la actividad [...] podemos ir [...] construyendo, [...] en las Enfermerías, en el Hospital General [...] no tiene un setting, [...] es todo muy flexible $[. .$.$] es mucho creado, [...] es$ tan potente por eso mismo [...]" (Mariana)

Este trabajo en el hospital parece exigir una deconstrucción de lo que es habitual para la construcción de algo nuevo, de nuevas posibilidades de creación.
"[...] yo comencé el servicio de Hematología, [...] era una gran novedad y fue un gran aprendizaje, y no tenía mucha regla, [...] vamos haciendo también por lo que vamos sintiendo en la relación [...]. Fue difícil para mí, porque era así como si tuviese que descubrir algo para construir otra cosa, [...]" (Tatiane)

"Pero yo creo que esa clínica diferenciada, [...] nueva y creo que posibilita tener más libertad para experimentar y arriesgar más, [...]" (Luiza)

De esta manera, al mismo tiempo en que el contexto hospitalario parece ofrecer muchas posibilidades de creación para esas profesionales, también es sentido por ellas como si allí no fuera su lugar. El discurso público de sus acciones no siempre fue fácil y estaba conquistado.

"[...] ahora yo creo que es difícil [...] apropiarse de que es posible hacer eso $[\ldots]$ " (Tatiane)

En la Hematología, principalmente, porque [...] todo el tiempo que está en juego es la cuestión de la infección, [...] eso es muy difícil, [...] como en los materiales, en las cosas, [...] a veces parece que hasta vos estás haciendo algo errado, [...]" (Mariana)

Mattingly (1998) al discutir la terapia ocupacional como el "ritual del cotidiano" actuado en el mundo no-cotidiano de la clínica, presenta esa cierta excentricidad. Las terapeutas ocupacionales de nuestra investigación resaltaron ese universo - de enfermedades, asepsias, sondas, problemas - en el cual no son conocidas, se da como una tensión, que no paraliza su trabajo, pero que se hace presente en su razonamiento.

"[...] una llega allá al hospital [...] no se está acostumbrada, las personas no están acostumbradas a nosotros, y ahí llegamos con la maleta, sin saber dónde poner, donde acostarse, ¿dónde se va a hacer grupo?" (Mariana)

"¿Que es lo que lleva en esa maleta', [...]?" (Luiza)

Acciones no centradas en el biomédico. 
"No tiene mesa, no tiene [...]" (Mariana)

"[...] hace aquel asunto de comer." (Luiza)

"[...] yo creo que el razonamiento clínico es lo mismo [...] los instrumentos son los mismos, pero [...] el espacio físico es otro, las cuestiones de aquel espacio son otras, [...] aquel no es un espacio [...] propiamente de la Terapia Ocupacional, [...] es la habitación de un paciente que está internado a toda hora entra una enfermera y hace procedimientos [...]" (Mariana)

Otra tensión vivida en la asistencia de terapia ocupacional en el ambiente hospitalario fue el tiempo cronológico, impuesto por la internación o por el desarrollo de la enfermedad, en contraposición al tiempo narrativo de la creación de historias con los pacientes, del proceso terapéutico. Para Mattingly (1998), el tiempo terapéutico es un tiempo narrativo, caracterizado por la vivacidad de los eventos y por su contribución para la trama que está siendo creada; cuya centralidad está en el actor, y no en la enfermedad y no su tiempo físico; gobernado por el deseo de que ciertos finales ocurren y no por cualquier trivialidad; es un tiempo de transformación; en que nada está garantizado; en que hay incertezas sobre lo que va a suceder.

"Y qué es lo que es el proceso [...] en esa clínica, ¿verdad? ¿Qué es lo que es el tiempo, qué es lo que es el proceso?" (Clarice, décimo segundo encuentro)

En el embate con el tiempo cronológico, las terapeutas ocupacionales contaron sobre su trabajo de construir narrativas con los pacientes que, en las palabras de Mattingly (1998), pudiesen ofrecerles posibilidades de encontrar algo deseable para el futuro. Las diferentes tramas compartidas pueden revelar un estilo altamente improvisado de trabajo, de procedimientos que pueden ser infinitos (Benetton, 2006) cuando son centrados en los sujetos. En este proceso pudieron ser nombrados varios procedimientos utilizados por ellas, como (a) la posibilidad de escoger hacer actividades menores para lidiar con el tiempo que se tiene de vida; (b) de ofrecer posibilidades de vivir y de construir alguna historia que tenga sentido aunque la muerte sea inminente o justamente por ese motivo, historia que puede ser narrada por las actividades realizadas con la terapeuta; (c) de despedirse por las actividades hechas con la terapeuta ocupacional; (d) de usar el cuerpo de la terapeuta para continuar en actividad.

Algunos procedimientos ya descritos en trabajos anteriores por Mastropietro, Oliveira \& Santos (2005 2008,2009 ) describen las posibilidades de los pacientes despiderense ante la conciencia de la muerte y de esa despedida ser comprendida como una actividad, al hacer actividades que permiten despedirse de las personas que gustan, que cuidaron de ellas, resolver pendientes, muchas veces subjetivos y emocionales.

"[...] es la primera vez que ella habla de morir, nunca tuvo eso, era siempre en el planeamiento de las actividades, el pedido de siempre de hacer las cosas para los otros, [...] ella misma [...] va disminuyendo, [...] antes ella hacía proyectos mayores, ahora ella pide para que yo haga sus uñas, l...l pregunta esa semana si tiene piecitas pequeñas de madera, quiere hacer cosas pequeñas, [...]" (Isadora)

"[...] porque tenía toda esa cuestión de todo el tiempo [...] no querer más intentar no querer más vivir, no querer hablar con nadie, [...] era de un modo muy hostil, [...] solo que en la terapia ocupacional en algunos momentos tenían [...] unos relámpagos de vida, [...] si yo salía y largaba la maleta allá con una actividad empezada, cuando yo volvía, ella estaba allá encima [...]" (Mariana)

"[...] cuando el grupo habla del tiempo [...] fui visualizando que la actividad va demarcando mucho [...] me veo [...] la imagen del empeoramiento muy marcada con la actividad. [...] el cuadro paro en tal momento que él no tenía más fuerzas para hacer, ahí la escritura paró en tal momento porque él no tenía más condiciones de continuar, porque entró en coma, [...] la actividad va demarcando mucho la vivencia del desmejoramiento." (Clarice)

"Yo recordé un caso que Fernanda atendió [...] una paciente que cada tanto quedaba con baja de plaquetas, [...] y [...] no puede [...] hacer ningún esfuerzo, y esa paciente 
[...]hacía mucho eso [...] de controlar el tiempo y 'entonces yo quiero aquella pequeña porque voy a terminar hoy, quiero hacer ahora, hoy' y cuando no alcanzaba igualmente ella quería hacer, entonces Fernanda iba haciendo y ella diciendo, [...] por las manos de Fernanda ella hablaba 'ahora voy a colocar la rosa, ahora voy a colocar la transparente' y [...] fue tan lindo,

[...]" (Mariana)

En estos dos encuentros, el grupo compartió muchas experiencias sobre la práctica y fue construido un sentido sobre el proceso terapéutico en terapia ocupacional y lo que es propio del hospital.

\section{DISCUSIÓN}

Al observar los elementos que dispararon tal proceso reflexivo en la CoP (y también en los diarios) podemos tener indicios de lo que fue considerado excepcional, fuera de lo usual, que demandó nuevas comprensiones, y que podría ofrecer indicios sobre la canonicidad de nuestra práctica.

Pero, ¿qué puede ser considerado canónico y no canónico en terapia ocupacional? Jerome Bruner (1997) sustenta que las acciones de los seres humanos son situadas en una cultura, en una matriz socialmente construida sobre cómo deberían ser las cosas (canonicidad), y que estos significados culturales orientan y controlan los actos individuales. Cuando se está delante de acontecimientos esperados, en ese sistema cultural, no hay necesidad de explicaciones. Sin embargo, delante de algo excepcional, imprevisible (no-canonicidad), surge la necesidad humana de ofrecer explicaciones narrativas que consigan establecer conexiones entre ese excepcional y el canónico, de modo que un sentido pueda ser construido para el sujeto - su interpretación, sus motivos.

La concepción de trabajo de la terapia ocupacional con proyectos de vida está vinculada a apuntar a una formación profesional pautada en un paradigma curativo, factor que dificulta acciones de cuidado con pacientes terminales. Los cursos de grado del área de la salud presentan carencia de asignaturas que aborden temas sobre la muerte, luto y de morir, factor que impacta directamente en la dificultad profesional de manejar situaciones en las cuales la cura no es posible (Santos, Corral-Mulato \& Bueno, 2014; Barbosa \& Carvalho, 2016).
La falta de capacitación profesional para trabajar en la clínica de la terminalidad implica una falta de visualización en la terapia ocupacional de que proyectos de vida sobrepasan todas las etapas del ciclo vital, y miedos, ansias, pendientes y deseos están presentes en el proceso de finitud y sustentan construcciones de proyectos de fin de vida. En ese sentido, los cuidados paliativos vienen siendo propagados con estrategias para intervenciones en terapia ocupacional (Baltazar, Pestana \& Santana, 2016), no obstante como todavía son prácticas y conocimientos incipientes, se reflejan como no canónicos en el cuidado de individuos fuera de la perspectiva de cura.

Se percibe que la temporalidad de los tratamientos y el setting terapéutico en el ámbito hospitalario son variables que ocasionan dinámicas poco habituales para el terapeuta ocupacional, pues el flujo asistencial abarca la evaluación de demandas e intervenciones delimitadas para internación, centradas en acciones de corto plazo y alta condicionadas al médico. Además, el sujeto no dirige la demanda del profesional, pero es la (el) terapeuta ocupacional quien va a su encuentro, estando más sujeto a rechazos del paciente a ser atendido en aquel momento, a la inestabilidad resultante de varios factores relativos al contexto hospitalario, bien como a la necesidad de adaptar espacios compartidos para construir un espacio objetivo-subjetivo para la atención (setting terapéutico).

En ese sentido, los principales elementos que promovieron la conversación sobre la terapia ocupacional deseada y practicada fueron los aspectos no-canónicos de la práctica: el espacio físico de trabajo, el tiempo de atención y la posibilidad inminente de la muerte en el hospital general. Tales aspectos explicitan la tensión vivida entre una práctica narrativa y el contexto necesario para el cuidado de las enfermedades.

En ese lugar, los pacientes se encuentran alejados de su vida cotidiana, y esa separación es institucionalmente marcada y aumentada por el universo no-común de la clínica en la que se encuentran confinados (Mattingly, 1998). El(la) terapeuta ocupacional, al estar atento/a las habilidades, a los proyectos, a las actividades, que se les brinda a los pacientes, a los cuales la muerte es inminente, tiene menor posibilidad de distanciarse de ellos en relación a personas, con historias de vida y proyectos que van a quedar inacabados.

Al nombrar algunos procedimientos utilizados por las terapeutas ocupacionales de la investigación junto a pacientes terminales, y compararlos a los trabajos de Mastropietro, Santos y Oliveira (2005, 2008, 2009), fue posible percibir que muchos de ellos ya estaban descritos por estos autores. Así mismo, ellos fueron narrados por las 
terapeutas ocupacionales de la investigación, iniciantes y experimentados, como construcciones en el improviso centradas en las necesidades de los pacientes y en las creencias de que el proceso terapéutico es construido en la relación.

No obstante haya evidencias vinculadas a la necesidad de mejorías en el proceso de formación profesional, en especial en relación a la finitud humana, el hecho ya citado parece corroborar con la proposición de que, a colocar la terapia ocupacional como objeto de estudio podemos llegar a algunas conclusiones que ofrezcan mejores sustentaciones para la práctica; conclusione que pueden ser revistas, re-hechas, ampliadas, en la medida en que la reflexión sobre los fenómenos de la práctica nos posibilite el acceso a nuevas indagaciones.

Mattingly (1998) también identificó aspectos de la terapia ocupacional que se asemejan al ritual de cura principalmente la posibilidad de transformación a través de una jornada. Asimismo, resaltó cuanto esta jornada está insertada en un universo que transita entre lo técnico y lo simbólico, en que las acciones de los terapeutas no invocan lo sagrado, al contrario, incluyen el cotidiano y son construidas en el improviso, en el suspenso de no conocer el próximo paso, pues él depende de la lectura de la respuesta del otro, de los sentidos que parecen estar siendo construidos.

\section{CONSIDERACIONES FINALES}

Este artículo pretendió elucidar cómo la tensión con el modelo biomédico se hizo presente, en una CoP, en las construcciones de sentido sobre la asistencia de terapeutas ocupacionales principiantes y experimentado en el contexto hospitalario. La huella recorrida tuvo inicio al compararse la práctica de terapia ocupacional en el contexto hospitalario con la que ocurre en otros contextos y explicitar que las diferencias en relación al tiempo terapéutico, al setting y a la posibilidad inminente de la muerte (en oposición a los proyectos de vida) son ncluidas en el razonamiento clínico, como cuestiones pragmáticas importantes y como variables relevantes para reflexiones en cuanto al proceso de formación profesional.

Así, al adentrar en el mundo clínico y no-cotidiano del hospital, en la medida en que ocurre la despersonalización de los sujetos, la terapia ocupacional es Ilamada a ofrecer posibilidades de vida, favoreciendo con que los pacientes se ubiquen de modo diferente en el hospital y consigan ocupar el tiempo vacío y común de la espera po procedimientos, con su creatividad personal (Benetton 2010). Además, el cuidado clínico de la enfermedad es favorecido(a) por la mejor disposición de los pacientes, (b) por la construcción- que puede ser compartida con otras personas del equipo - de una imagen de una persona que hace, piensa y tiene una historia de vida, que no solamente ocupa el lugar de enfermo, (c) por la mejor posibilidad de manejo del dolor y de los procedimientos hospitalarios.

En ese camino, fue posible identificar procedimientos de terapia ocupacional para pacientes de la clínica de la terminalidad, como(a) la posibilidad de escoger hace actividades menores para lidiar con el tiempo que se tiene de vida; (b) de ofrecer posibilidades de vivir y de construir alguna historia que tenga sentido aunque la muerte sea inminente o justamente por ese motivo, historia que puede ser narrada por las actividades realizadas con la terapeuta; (c) de despedirse por las actividades hechas con la terapeuta ocupacional; (d) de usar el cuerpo de la terapeuta para continuar en actividad.

De ese modo, el ejercicio de investigar la práctica y de compartirla en un espacio formativo, como la $\mathrm{Co}-$ munidad de Práctica (Marcolino et al, 2016; Galheigo et al, 2015; Loughan, 2010; Wenger, 1998) favoreció la apropiación del razonamiento clínico a partir de las particularidades de una práctica de terapia ocupacional en contexto hospitalario.

Como se trata de una investigación-acción, nuestros resultados presentan limitaciones en cuanto a sus posibilidades de generalización. De ese modo, esperamos que el artículo pueda contribuir para una mejor comprensión de las características de la práctica en terapia ocupacional en los contextos hospitalarios, así como para la mejoría de la formación inicial y continuada, además de estimular cuestiones de investigación en esa temática. 


\section{REFERENCIAS BIBLIOGRÁFICAS}

Angeli, A. A. C.; Luvizaro, N. A.; Galheigo, S. M. (2012). O cotidiano, o lúdico e as redes relacionais: a artesania do cuidar em terapia ocupacional no hospital. Interface - Comunicação, Saúde, Educação, 16(40), 261 272. Epub April 19, 2012. DOI:10.1590/S1414-32832012005000016

Angerami-Camon, V. A. (2010). O Psicólogo no hospital. In: Angerami-Camon, V. A. (org.) Psicologia Hospitalar: teoria e prática $2^{a}$ edição revista e ampliada (2ed., pp.2-3). Cengage Learning.

Álvarez, E., Gallegos, S., Romero, E., Moraga, C.; López, M.; Estalleda, C., Viviani, V., et al. (2011) Impacto de intervención temprana a cuidadores de pacientes secuelados de un accidente cerebro vascular. Estudio piloto. Revista Chilena de Terapia Ocupacional, 11 (2), 1-16.

Baltazar, H. M. C.: Pestana, S. C. C.; Santana, M. M. R. (2016). Contributo da intervenção da terapia ocupacional nos cuidados paliativos. Cadernos de Terapia Ocupacional UFSCar, 24(2), 261-273.

Barbosa, L. H.; Carvalho, I. S. (2016). A perspectiva de residentes sobre a morte e seu reflexo na relação com os pacientes. Revista da SBPH 19(2), 107-128.

Bardin, L. (2011) Análise de Conteúdo. Lisboa/Portugal: Edições 70.

Benetton, M. J. (2006). Trilhas Associativas: ampliando subsidios metodológicos à clínica da terapia ocupacional. Campinas Brasil: Arte Brasil Editora / UNISALESIANO - Centro Universitario Católico Auxilium.

Benetton, M. J. (2010). O encontro do sentido do cotidiano na Terapia Ocupacional para a construção de significados. Revista CETO, 12(12), 32-39.

Briceño, C. (2011). Reflexiones en torno al razonamiento bioético en cuidados paliativos. Revista Chilena de Terapia Ocupacional, 11 (2), 1-13. DOI: 10.5354/0717-5346.2011.17777

Bruner, J. (1997). Atos de significação. Porto Alegre: Artes Médicas.

Desroche, H. (2006). Pesquisa-ação: dos projetos de autores aos projetos de atores e vice-versa. (33-68), en Thiollent, M. Pesquisa-ação e Projeto Cooperativo na Perspectiva de Henri Desroche. São Carlos Brasil: EDUFSCar.

Galheigo, S. M.; Braga, C. P.; Mieto, F. S. R.; Viotti, F.; Sarmento, G. Q.; Motta, M.; Silva, M. C. et al. (2015). Comunidade de prática em terapia ocupacional: a avaliação do processo pelos participantes e pelos pesquisadores. Cadernos de Terapia Ocupacional da UFSCar, 23 (3), 463-474. DOI: 10.4322/0104-4931.ctoAO047

Grossman, P., Winengurg, S., Woolworth, S. (2001). Toward a Theory of Teacher Community. Teachers College Record, 103 (6), 942-1012.

Marcolino, T. Q., Fantinatti, E. N., Forneretto-Gozzi, A. P. N., Cid, M. F. (2016) Comunidade de prática em terapia ocupacional para o cuidado em saúde mental na atenção básica em saúde: expectativas e impactos. Cadernos de Terapia Ocupacional da UFSCar, 24 (4), 733-741.

Mastropietro, A. P.; Oliveira, E. A.; Santos, M. A. (2005). Intervenções do terapeuta ocupacional em um caso de terminalidade: Associações Finais. Revista CETO, 1, 18-28.

Mastropietro, A. P., Oliveira, E. A.; Santos, M. A. (2008). Clínica da Terminalidade. Revista CETO, 11, 18-25.
Mastropietro, A. P.; Oliveira, E. A · Santos, M. A · Voltarellii, J. C. (2009), Terapia Ocupacional. (337-346), en Machado, L. N.; Camandoni, V. O.; Leal, K. P. H.; Moscatello, E. L. M. Transplante de Medula Óssea: Abordagem Multidisciplinar. São Paulo Brasil: Lemar.

Mattingly, C. (1998). Healing dramas and clinical plots: the narrative structure of experience. Cambridge: Cambridge University Press.

Mattingly, C., Fleming, M. H. (1994). Clinical Reasoning: forms of inquiry in a therapeutic process. Philadelphia: F. A. Davis Company.

Palm, R. C. M. (2016). Terapia Ocupacional en Contextos Hospitalarios. TOG (A Coruña) [revista en internet]. [03/08/2016]; 13 (23), [12p.]. Disponible en: http://www.revistatog.com/num23/pdfs/editorial.pdf

Pierre, B. L. (2009). Occupational Therapy as Documented in Patients Records Part III. Valued but not Documented. Underground Practice in the Context of Professional Written Communication. Scandinavian Journal of Occupational Therapy. 8 (4), 174-183.

Preece, J. (2004). Etiquette, empathy, and trust in communities of practice: stepping-stones to social capital. Journal of Universal Computer Science. 10 (2), 294-302. doi:10.3217/jucs-010-03-0294.

Marcolino, T. Q., Reali, A. M. M. R. (2012). Rotas dissonantes e comunidade profissional: pistas para promover a aprendizagem colaborativa. En Realli, A. M. M. R. (Org.). Desenvolvimento profissional da docência: teorias e práticas. São Carlos: Edufscar.

Marcolino, T. Q.; Reali, A. M. M. R. (2016). “Crônicas do Grupo: ferramenta para análise colaborativa e melhoria na reflexão na pesquisa-ação. Interface (Botucatu). 20 (56), 65-76. doi:10.1590/1807-57622015.0257.

Marcolino, T. Q.; Ferreira, G. L.; Reali, A. M. M. R. (2017). “Isso eu levo para a vida": aprendizagem da prática profissional em uma Comunidade de Prática. Interface (Botucatu). 21 (61) 411-420. doi:10.1590/180757622016.0099

Riffo, M. A. R. (2013). Terapia Ocupacional en Hospital General de Agudos de la ciudad autónoma de Buenos Aires. Argentina. Revista Chilena de Terapia Ocupacional, 13 (2), 99-112.

Robertson, L. (2012.) Clinical reasoning in Occupational Therapy: controversies in practice. West Sussex: Wiley-Blackwell.

Santos, J. L.; Corral-Mulato, S.; Bueno, S. M. V. (2014). Morte e luto: a importância da educação para o profissional de saúde. Arq. Cienc. Saúde UNIPAR, 18(3), 199-203.

Schell, B. A. B.,Schell, J. W. (2008). Clinical and Professional Reasoning in Occupational Therapy. Philadelphia: Wolters Klumer/Lippincott Williams \& Wilkins Publishers.

Toledo, R. F., Giatti, L. L., Jacobi, P. R. (2014). A pesquisa-ação em estudos interdisciplinares: análise de critérios que só a prática pode revelar. Interface: Comunicação, Saúde, Educação, 18 (51), 633-646. DOI: 10.1590/1807-57622014.0026

Wenger, E. (1998). Communities of practice: learning, meaning and identity. Cambridge: Cambridge University Press.

Wesley, P., Buysse, V. (2001). Communities of practice: expanding professional roles to promote reflection and shared inquiry. Topics in Early Childhood Education. 21 (2), 114-123. DOI: 10.1177/027112140102100205 